\title{
Frecuencia de granuloma periapical y quiste radicular diagnóstico histopatológico posextracción dental en la Facultad de Odontología, UNAH
}

\author{
David Sierra ${ }^{1}$ \\ Ricardo Aguilar ${ }^{2}$
}

\section{RESUMEN}

El objetivo de esta investigación es identificar la frecuencia y ubicación del granuloma periapical y quiste radicular realizando un estudio histopatológico posextracción dental en la Facultad de Odontología de la UNAH. Para lograrlo se recolectaron 97 lesiones periapicales con criterio de exodoncia indicada por estudio radiográfico con destrucción ósea periapical mayor de $1.5 \mathrm{~mm}$; dichas biopsias fueron fijadas en formalina buferada, a las cuales se les realizó un estudio histopatológico.

Los datos se tomaron de las historias clínicas y fichas de registro realizadas a los pacientes después de extraer el órgano dental afectado, posteriormente se introdujeron los datos al programa estadístico SPSS versión 19 para su análisis.

Los resultados indican que el diagnóstico más frecuente corresponde al quiste radicular con el $53.6 \%$, el género femenino es el más afectado con un $55.6 \%$, en la tercera década de vida hay una mayor presencia de quiste radicular en un $18.5 \%$ de las 97 muestras. Esta investigación proporciona información al personal de odontología para la detección, diagnóstico, manejo y seguimiento de las lesiones periapicales.

Palabras clave: quiste periapical, granuloma periapical, histopatológico.

\section{ABSTRACT}

Objective: To identify the frequency and location of periapical granuloma and cyst Root histopathology making a post - tooth extraction Dental School UNAH. Methodology: 97 periapical lesions were collected with extraction criteria indicated

\footnotetext{
${ }^{1}$ Beneficiario de una beca básica de la DICYP. Estudiante de Odontología, Facultad de Odontología, UNAH: Dasierra4@gmail.com.

${ }^{2}$ Asesor, docente, Departamento de Estomatología, Facultad de Odontología, UNAH.
} 
by radiographic study more periapical bone destruction $1.5 \mathrm{~mm}$, these buffered formalin-fixed biopsies which were performed a histopathological study data were taken from medical records and registration cards on to after removing the dental organ affected patients subsequently entered data to SPSS version 19 for analysis. Results: The most frequent diagnosis Radicular cyst corresponds to the $53.6 \%$, the female affected with $55.6 \%$ in the third decade of life there is a greater presence of radicular cyst $18.5 \%$ of the 97 samples. Conclusions: This research will provide information to the dental personnel for the detection, diagnosis, management and follow-up of periapical lesions

Keywords: periapical cyst, periapical granuloma, histopathology.

\section{INTRODUCCIÓN}

Las lesiones periapicales (LP) son las patologías más frecuentes de origen odontogénico resultado de la necrosis pulpar (Carrillo, Vera, Peñarrocha y Martí, 2007). Es la descomposición séptica del tejido conjuntivo pulpar que ocasiona destrucción del sistema microvascular y linfático, disminución del drenaje de líquido inflamatorio y aumento de presión de los tejido. Esto conlleva a la común de las LP, el granuloma periapical, que fundamentalmente es una masa compuesta por tejido de granulación, principalmente macrófagos, linfocitos y célula plasmáticas; estas células pueden contener cuerpos de Russell, muchos fagocitos ingieren material lípido, agrupándose, y forman las llamadas células espumosas (ver figura 1).

El quiste radicular de origen odontogénico se forma en el periápice de un diente con pulpa necrótica, se considera generalmente secuela directa de un granuloma (Kumar, Achuthan, Logan y Augustine, 2013); derivan de los restos epiteliales de Malassez originados en los vestigios de la vaina de Hertwig (Lin, Huang y Rosenberg, 2007). Este epitelio prolifera por el estímulo inflamatorio formando una cavidad patológica llena de líquido, revestida por epitelio de tipo escamoso, estratificado de grosor variable y ares con abundancia de células inflamatorias (Shafer y Levy, 1986), (ver figura 2).

La presencia de estas lesiones sobrelleva a la resorción ósea causada por patógenos, expresando una serie de posibles factores de virulencia, tales como toxinas, proteasas, adhesinas e lipopolisacárido, que contribuyen a la licuefacción del tejido alterando la función de los osteoblastos y osteoclastos, que son células especializadas para la formación y mantenimiento óseo. Los osteoblastos producen proteínas de la matriz del hueso y se hacen cargo de la mineralización de los tejidos, mientras que los osteoclastos son células multinucleadas responsables 
de la resorción ósea, manteniendo así un equilibro homeostático del tejido (Koide, Kinugawa, Takahashi y Udagawa, 2010).

Figura 1. Granuloma periapical

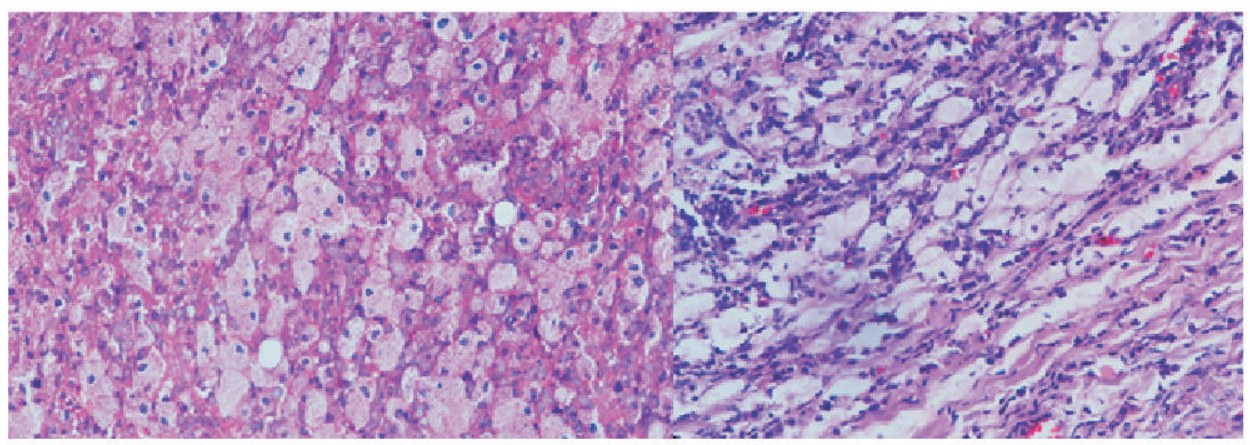

Microfotografía que muestra aspectos histológicos $(\mathrm{A})$ células espumosas conteniendo material mucoide (B) rodeado por una cápsula de tejido conectivo.

Las lesiones periapicales se manifiestan como una radiolucidez, lo cual es la consecuencia de la destrucción ósea que conlleva el proceso inflamatorio crónico (Segura, Sánchez y Calvo, 2010), ocasionando un tejido de granulación (neoformación sanguínea, proliferación de fibras colágenas e infiltrado celular) que puede alcanzar un tamaño aproximado de 2 a $5 \mathrm{~mm}$ de diámetro, lo cual puede ser un indicio de un granuloma periapical (Duarte, et al, 2007).

La imagen radiográfica de los quistes periapicales es radilúcida con forma redondeada u ovoide, borde bien delimitado radiopaco delgado, continuo a la lámina dura del diente, circunscrita y en el ápice de un diente desvitalizado ambas lesiones presentan similares características radiográficas; en consecuencia, tratar de realizar un diagnóstico clínico entre granuloma y quiste es casi imposible (Wood, 1984), pero si la lesión es muy grande, es más probable que sea un quiste y no un granuloma periapical (González, Moret y Jiménez, 2011).

Pero para alcanzar una tamaño considerable tiene que trascurrir un periodo de tiempo y se pueden generar otros inconvenientes, es decir, la complicación por la presencia de LP es muy amplia, desde un quiste radicular, neoplasias benignas y malignas, lesiones óseas; enfermedades sistémicas hasta una de las más álgidas, como el carcinoma intraóseo primario, que es un tumor maligno derivado de tumores odontogénicos que se presenta exclusivamente en los maxilares, su desarrollo proviene de los restos epiteliales de Malassez involucrados en la odontogénesis. 
La Organización Mundial de la Salud (OMS) sugirió el término de carcinoma intraóseo primario y catalogó la lesión como un carcinoma odontogénico, según clasificación 2005 - OMS (Tarakji, Shireen, Umair, Nasser, Alzoghaibi \& Hanouneh, 2013).

Existen publicaciones en el extranjero de estudios similares, pero no se ubicaron investigaciones en Honduras que involucren un análisis cuantitativo o cualitativo de estas patologías en particular, para un mejor control y manejo en beneficio de la población.

En síntesis, la finalidad de este proyecto es determinar la frecuencia del granuloma periapical y el quiste radicular, analizar variables como edad, género y región anatómica y compararlas con otras publicaciones de distintas áreas geográficas. Esto proporcionará información a estudiantes, profesionales y docentes para una aproximación, diagnóstico, pronóstico y tratamiento oportuno para la obtención de mejores resultados y seguimiento de dichas lesiones.

Figura 2. Quiste periapical revestido por epitelio de tipo escamoso, estratificado de grosor variable y ares con abundancia de células

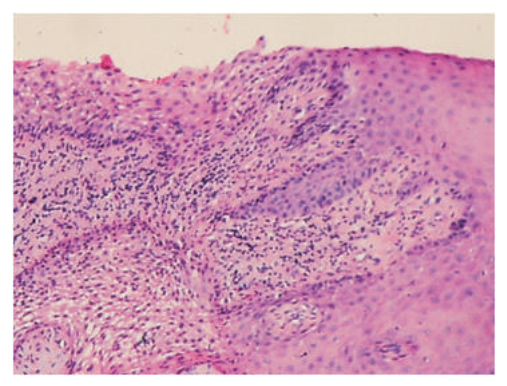

\section{METODOLOGÍA}

Se realizó un estudio transversal descriptivo, con muestra de n (97) pacientes que presentaron lesiones periapicales, detectadas radiográficamente y con sus respectivos reportes histopatológicos. Los participantes en el estudio acudieron en un tiempo determinado de diez meses a la clínica de cirugía bucal de la Facultad de Odontología.

La recolección de los datos se hizo a través de historias clínicas con sus respectivas radiografías periapicales, consentimiento informado y una ficha de registro como instrumento de investigación para variables como edad, género, localización anatómica en la cavidad oral y su relación con el diagnóstico histopatológico; 
previamente el procedimiento quirúrgico que consistía en la extracción dental y biopsia excisional. Con una población de $\mathrm{N}=130$ lesiones periapicales extraídas a pacientes, la muestra se calculó con un coeficiente de confianza del $95 \%$ y un margen de error de un $5 \%$; se utilizó el programa estadístico decisión Analist 2.0 y el tipo de muestreo fue aleatorio simple o al azar.

Las lesiones periapicales extraídas se sumergieron inmediatamente en formalina buferada al menos durante 48 horas, seguidamente la desmineralización en una solución de $22,5 \%$ de ácido fórmico y $10 \%$ de citrato de sodio durante un período de 3 a 4 semanas con agitación constante. Después de enjuagar de 24 a 48 horas en agua corriente, las muestras se deshidrataron y se procesaron para el corte histológico de rutina, la muestra fue incrustada en parafina en su totalidad. Se tomaron con el micrótomo fijado en 4 a $5 \mu \mathrm{m}$ hasta que toda la muestra fue cortada, los portaobjetos se tiñeron con hematoxilina y eosina.

Se evaluaron las lesiones bajo microscopio de luz, las lesiones inflamatorias fueron diagnosticadas y clasificadas de acuerdo a lo siguiente: granuloma periapical inflamación granulomatosa con predominio linfocitico, células plasmáticas y macrófagos. Estas lesiones pueden ser epitelializadas o no epitelializadas (Carrillo, Vera, Peñarrocha y Martí, 2007). Quistes periapicales, cavidad patológica con un revestimiento epitelial puede estar o no en comunicación con el canal radicular (Lin, Huang \& Rosenberg, 2007). Obtenido el diagnóstico histopatológico se introdujeron los datos electrónicamente, se utilizó el programa SPSS versión 19, para el análisis de correlación de variables se elaboró una tabla.

Aspectos éticos: cada paciente que participó en el proyecto de investigación fue incluido por medio de un consentimiento informado. Los criterios de exclusión fueron: pacientes menores de 15 años y mayores de 50 años de edad, terceros molares.

\section{RESULTADOS}

Los granulomas y quistes periapicales son patologías dentales que se producen como consecuencia de la necrosis pulpar, por lo tanto, son consideradas de naturaleza inflamatoria (Brave, Madhusudan, Ramesh y Brave, 2011). Durante el periodo de esta investigación se obtuvo una muestra de 97 lesiones periapicales, a las cuales se les realizó un estudio histopatológico, de las cuales 52 muestras correspondieron a quiste radicular con $53.6 \%$ y 45 a granulomas periapicales 46.4 $\%$ (ver tabla 1). 
Tabla 1. Frecuencia de quiste radicular y granuloma periapical

\begin{tabular}{|l|c|c|}
\hline \multicolumn{1}{|c|}{ Diagnóstico } & Frecuencia & Porcentaje \\
\hline Quiste radicular & 25 & $53.6 \%$ \\
\hline Granuloma periapical & 45 & $46.4 \%$ \\
\hline Total & 97 & $100 \%$ \\
\hline
\end{tabular}

Grafico 1. Frecuencia de quiste radicular y granuloma periapical

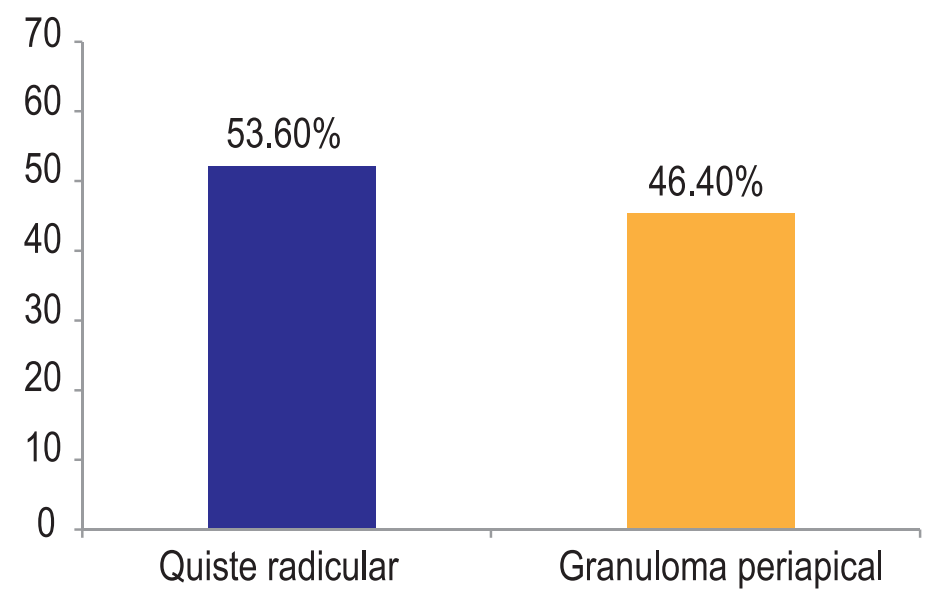

Tabla 2. Distribución de presencia de lesiones periapicales con respecto al género

\begin{tabular}{|l|c|c|}
\hline \multicolumn{1}{|c|}{ Género } & Frecuencia & Porcentaje \\
\hline Masculino & 43 & $44.3 \%$ \\
\hline Femenino & 54 & $55.7 \%$ \\
\hline Total & 97 & $100 \%$ \\
\hline
\end{tabular}

La tabla 2 muestra la distribución de lesiones periapicales con respecto al género con un $55.7 \%$ para el género femenino y un $44.3 \%$ el género masculino. El quiste radicular es el diagnóstico de mayor frecuencia entre los pacientes a los cuales se les realizó una extracción dental y las mujeres son más perjudicadas por la presencia de lesiones periapicales en comparación a los hombres. 
Tabla 3. Distribución de quiste radicular y granuloma periapical con respecto al género

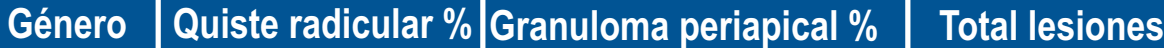

\begin{tabular}{|l|c|l|l|} 
Masculino & $25(25.7 \%)$ & $18(18.5 \%)$ & 43 \\
\hline Femenino & $27(27.8 \%)$ & $27(27.8 \%)$ & 54 \\
\hline Total & $\mathbf{2 5}$ & $\mathbf{4 5}$ & $\mathbf{9 7}$
\end{tabular}

Grafico 2. Distribución de quiste radicular y granuloma periapical con respecto al género

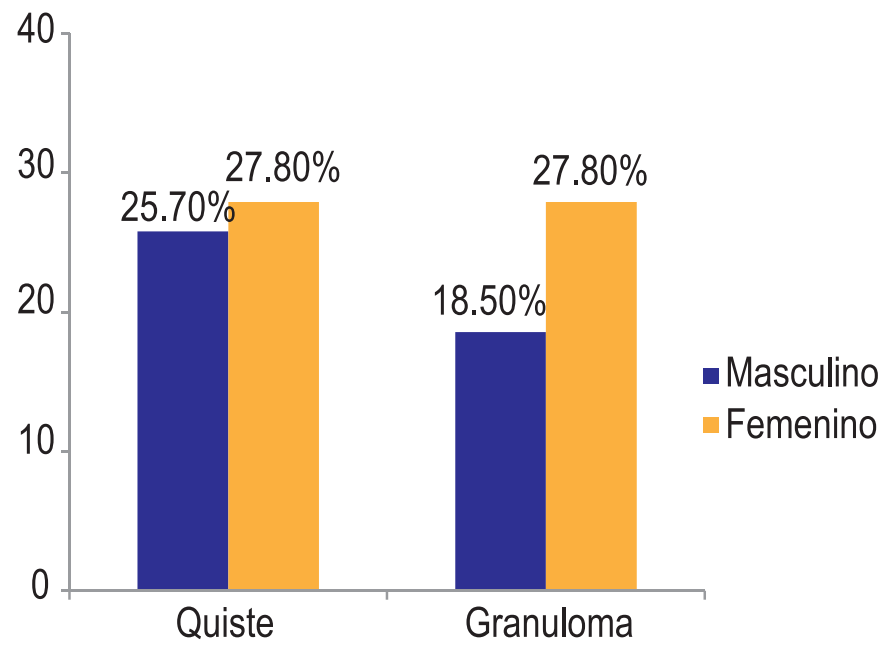

En la tabla 3 se observa la distribución de quiste radicular y granuloma periapical con respecto al género, en donde el género femenino presenta la mayoría de quistes radiculares; al igual que los granulomas periapicales, una relación entre la tabla 2 y la tabla 3 refleja que el género femenino es el más afectado en las lesiones periapicales y en ambos diagnósticos.

De igual forma, se analizó la presencia de lesiones periapicales con respecto a la década de vida, dando como resultado lo siguiente: segunda década de vida (11-20 años ) con el $16.5 \%$, tercera década de vida (21-30) $29.9 \%$, cuarta década de vida (31-40) $21.6 \%$, quinta década de vida (41-50) $11.3 \%$, sexta década de vida (51-60) $15.5 \%$, séptima década de vida (61-70) 5.1\%; siendo la más perjudicada la tercera década de vida. En la tabla 4 se contempla la distribución de década de vida y el diagnóstico histopatológico. 
Tabla 4. Distribución de décadas de vida y diagnóstico histopatológico

\begin{tabular}{|l|c|c|c|}
\hline \multicolumn{1}{c|}{ Edades } & Quiste periapical & Granuloma periapical & Total \\
\hline 11 - 20 años & $7(7.2 \%)$ & $9(9.2 \%)$ & $16(16.5 \%)$ \\
\hline $21-30$ años & $18(18.5 \%)$ & $11(11.3 \%)$ & $29(29.9 \%)$ \\
31 - 40 años & $10(10.3 \%)$ & $11(11.3 \%)$ & $21(21.6 \%)$ \\
$41-50$ años & $7(7.2 \%)$ & $4(4.1 \%)$ & $11(11.3 \%)$ \\
$51-60$ años & $9(9.2 \%)$ & $6(6.1 \%)$ & $15(15.5 \%)$ \\
$61-70$ años & $1(1.03 \%)$ & $4(4.1 \%)$ & $5(5.1 \%)$ \\
\hline Total & $\mathbf{5 2}$ & $\mathbf{4 5}$ & $\mathbf{9 7}$
\end{tabular}

Grafico 3. Distribución de décadas de vida y diagnóstico histopatológico

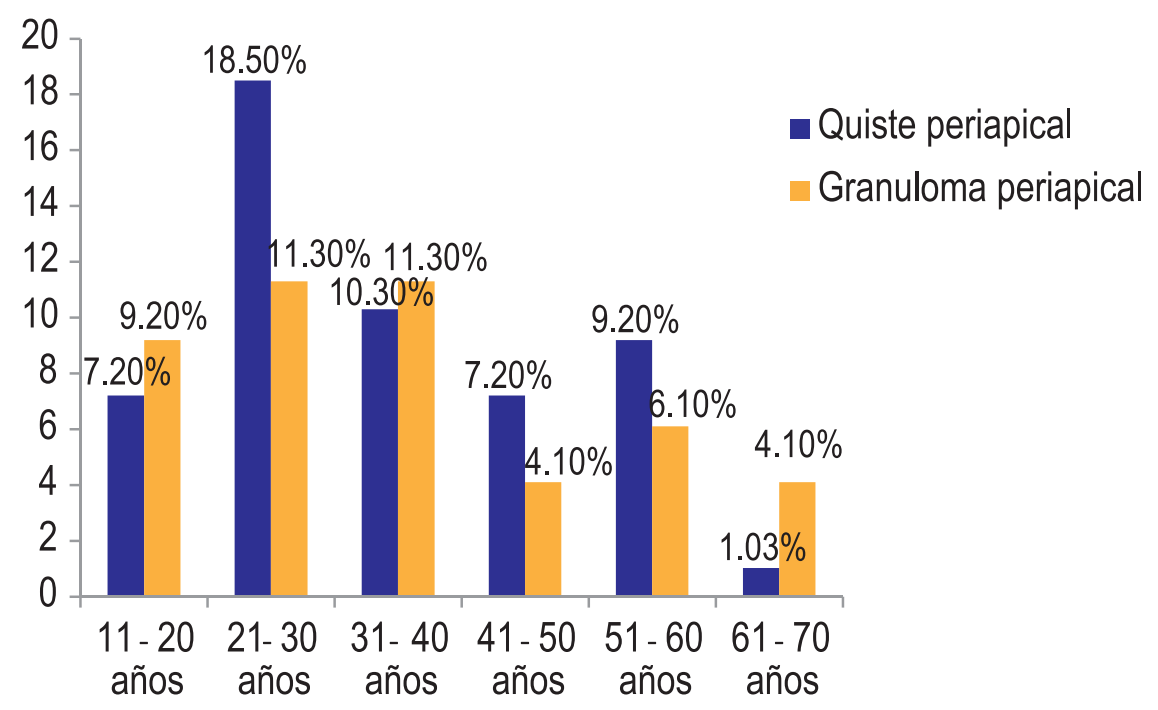

Los pacientes entre 21 a 30 años son los más afectados, esto indica que a los pacientes entre estas edades que se les realiza una extracción dental y se observa lesión periapical, la probabilidad de que sea un quiste radicular es mayor en comparación a un granuloma periapical.

Para analizar la región anatómica con mayor presencia de lesiones periapicales, se dividió la cavidad oral en cuatro cuadrantes, obteniendo como resultados: superior derecho $18.5 \%$, superior izquierdo $24.7 \%$, inferior izquierdo $26.8 \%$, inferior derecho $29.9 \%$. En la tabla 5 se analiza la distribución de la región anatómica y diagnóstico histopatológico. 
Tabla 5. Distribución de la región anatómica y diagnóstico histopatológico

\begin{tabular}{l|c|c|c|}
\hline \multicolumn{1}{c|}{ Cuadrante } & Quiste periapical & Granuloma periapical & Total \\
\hline Superior derecho & $10(10.3 \%)$ & $8(8.2 \%)$ & $18(18.5 \%)$ \\
\hline Superior izquierdo & $18(18.5 \%)$ & $6(6.2 \%)$ & $24(24.7 \%)$ \\
\hline Inferior izquierdo & $10(10.3 \%)$ & $16(16.5 \%)$ & $26(26.8 \%)$ \\
\hline Inferior derecho & $14(14.4 \%)$ & $15(15.5 \%)$ & $29(29.9 \%)$ \\
\hline Total & $\mathbf{5 2}$ & $\mathbf{4 5}$ & $\mathbf{9 7}$ \\
\hline
\end{tabular}

Gráfico 4. Distribución de la región anatómica y diagnóstico histopatológico

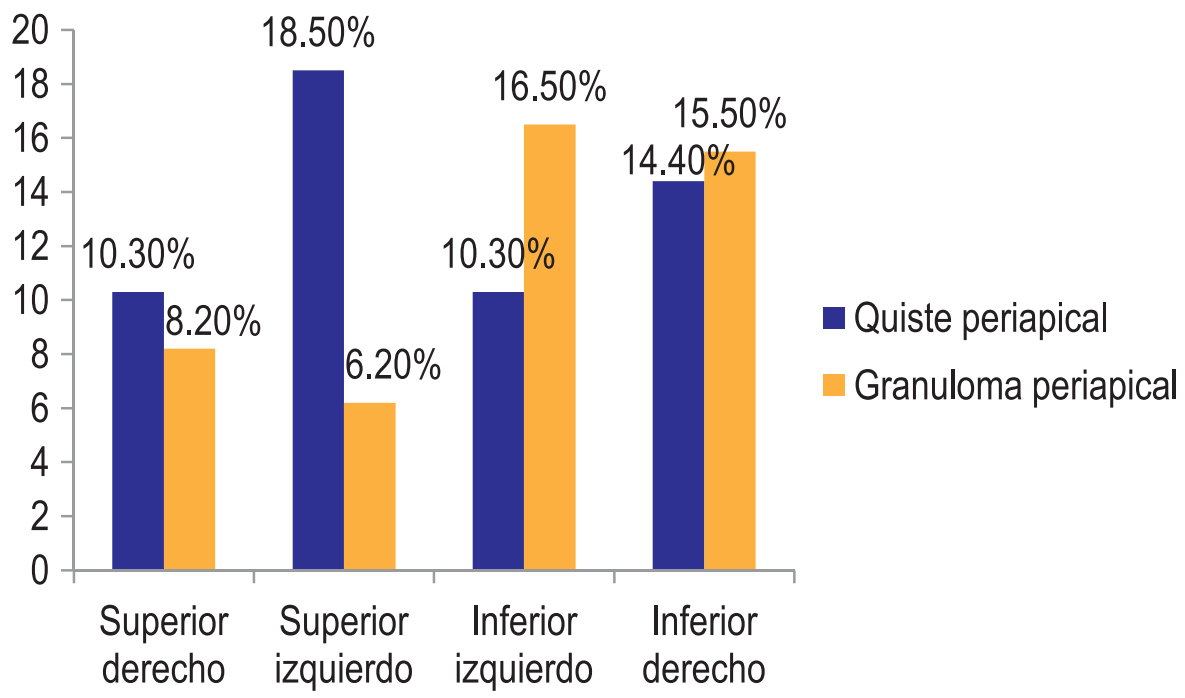

\section{DISCUSIÓN}

En nuestro estudio del total de muestras analizadas, la mayor frecuencia corresponde al quiste radicular con el $53.6 \%$ (52) y el granuloma periapical con 46.3 $\%$ (45). Difiriendo con el estudio realizado en Guatemala (Mena 1988), en el cual se percibe una mayor frecuencia de granuloma periapical con el $71.33 \%$ y el quiste radicular con $20.66 \%$.

La proporción de biopsias fue mayor para el género femenino con $55.6 \%$, con relación al masculino con $44.3 \%$. En otro estudio, Enríquez (2011) también observa una mayor proporción en mujeres con $65,0 \%$ y hombres con un 35,0\%. En nuestra investigación el granuloma periapical y el quiste radicular tienen una mayor frecuencia en el género femenino con un $27.8 \%$ para ambos diagnósticos, coincidiendo con los resultados de Mena. 
La etapa de vida más afectada es de 21-30 años (tercera) para el granuloma periapical con un $18.5 \%$ y el quiste radicular con $11.3 \%$ para un total de $29.8 \%$ (lesiones periapicales). Coincidiendo con (Enríquez 2011) y ( Mena 1988), la mayor distribución se da en la tercera y cuarta década.

El cuadrante anatómico de la cavidad oral más afectado por el quiste radicular fue el cuadrante superior izquierdo con un $18.5 \%$; en cambio, el cuadrante inferior izquierdo tuvo mayor frecuencia de granulomas periapicales con un $16.5 \%$. No existen estudios con el cual se puedan comparar las lesiones con respecto a la zona anatómica de la cavidad oral.

\section{CONCLUSIONES}

1. Tras el examen histopatológico realizado a 97 lesiones periapicales, el diagnóstico con mayor frecuencia corresponde al quiste radicular con el $53.6 \%$ y el granuloma periapical con $46.4 \%$.

2. Según los datos recolectados, se observa una mayor frecuencia de lesiones periapicales en el género femenino con el $55.7 \%$, en donde existe un distribución equitativa del $50 \%$ tanto para el quiste radicular como para granuloma periapical.

3. Según el diagnóstico histopatológico y la información recolectada en la tercera década de vida, se presentan más lesiones periapicales con el $29.8 \%$. Igualmente, es en la tercera década de vida donde existe la mayor frecuencia de quistes radiculares con el $18.5 \%$ y el de granulomas periapicales con $11.3 \%$.

4. La zona anatómica con mayor presencia de lesiones periapicales es el cuadrante inferior derecho con un $29.8 \%$, sin embargo, donde se observa una mayor presencia de quistes radiculares es en el cuadrante superior izquierdo con un $18.5 \%$ y el granuloma periapical con el $16.5 \%$ en el cuadrante inferior izquierdo, del total de 97 lesiones periapicales.

\section{BIBLIOGRAFÍA}

Brave, D.; Madhusudan, A.S; Ramesh, G. y Brave,V.R. (2011). Radicular cyst of anterior Maxilla. International Journal of Dental Clinics, 3(2), 16-17.

Carrillo, C.; Vera, F.; Peñarrocha, M. \& Martí, E. (2007). The post-endodontic periapical lesion:Histologic and etiopathogenic aspects. Med Oral Patol Oral Cir Bucal, 12(8). 
Duarte, E.; Vallejos, A.; Briend, M.; Quetglas, M.; Almiron, M. y Solís, M. (2007). Identificación de los restos epiteliales de Malassez en granulomas dentarios periapicales determinantes del diagnóstico. Revista Ateneo Argentino de Odontología, 46(2), 18 - 25.

Enríquez, F. (2011). Análisis de las lesiones periapicales de origen endodontico en paciente de la Facultad de Odontología de Tijuana, Baja California, México. Universidad Autónoma de Baja California, Granada, España. Recuperado de: http://0-hera.ugr.es.adrastea.ugr.es/tesisugr/1988803x.pdf

González, J.; Moret de González y Jiménez, L.(2011). Quiste periodóntico apical de gran tamaño en el maxilar. Reporte de un caso clínico. Acta Odontológica Venezolana, 3(49), 1-5.

Koide, M.; Kinugawa, S.; Takahashi, N. \& Udagawa, N. (2010). Osteoclastic bone resorption induced by innate immune responses. Periodontology 2000, 54, 235 246.

Kumar, A.; Achuthan, N.; Logan, K. \&Augustine, D. (2013). Effective Management of a Large Radicular Cyst Masanori Koide 201, Osteoclastic bone resorption induced by innate immune responses. Periodontology 2000 with Surgical Enucleation. Oral and Maxillofacial Pathology Journal, 5(1).

Lin, M.; Huang G.L. \& Rosenberg, P. (2007). Proliferation of Epithelial Cell Rests, Formation of Apical Cysts and Regression of Apical Cysts after Periapical Wound Healing. Journal of Endodontics, 33(8).

Mena, O. (1988). Lesiones periapicales en pacientes de clínicas privadas de Guatemala: correlación entre características clínicas e histopatológicas. Tesis. Universidad Francisco Marroquín.

Segura, J.; Sánchez, B. y Calvo,C. (2010). Medicina endodoncica:implicaciones sistemicas de la patologia y terapeutica endodoncica. Endodoncia, 28(4), 233240.

Shafer, W. y Leavy, B. (1986). Tratado de patología bucal. Enfermedades de la pulpa ylos tejidos periapicales. EE.UU.: Interamericana.

Tarakji, B.; Shireen, A.; Umair, A.; Nasser, S.; Alzoghaibi, I. \& Hanouneh, S. (2013). Malignant Transformation of Radicular Cyst/Residual Cyst: A Review of Literature. British Journal of Medicine \& Medical Research, 4(25).

Wood, N. (1984). Periapical lesions. Dental Clinics of North America. 